\title{
Capillary Phenomena at Grain Boundaries Gains Focus at HTC-5 www.sri.ua.es/congresos/htc2007
}

The 5th International Conference on High-Temperature Capillarity HTC-5 was held March 21-24, 2007 in Alicante, Spain under the auspices of the Instituto Universitario de Materiales de Alicante of the Universidad de Alicante. The conference was chaired by Enrique Louis of the Universidad de Alicante and the program was chaired by Nicolas Eustathopoulos of Polytechnic Institute of Grenoble, France.

Within a format of a single venue for all presentations, with no parallel sessions, the conference presented topics of fundamental science that ranged from the energetic and structural properties of surfaces and interfaces to wetting phenomena with a particular emphasis on the role of interfacial reactions in wettability and adhesion. Several presentations, from both invited and contributed speakers, were focused on a variety of processes in which capillarity plays an important and sometimes decisive role. For example, there were presentations on processing of metal-matrix composites by infiltration routes; joining of metals and ceramics by brazing, soldering, and welding; crystal growth of monocrys- talline semiconductors; and even processing of metallic foams.

The conference has confirmed and amplified a tendency started in the last HTC conference held at Sanremo, Italy in 2004, consisting in a significant increase of presentations on capillary phenomena at grain boundaries, a topic that has many important ramifications in materials science and engineering such as liquid-metal or grain-boundary embrittlement phenomena. The conference benefited from the participation of theoretical groups modeling problems such as the surface and interface energies and structures in relation with nanofriction, the influence of thermal fluctuations on the surface structure of free liquid surfaces, the adhesion energies of metals on ceramics and even the phase diagrams of nanosystems.

Important studies were also presented on new experimental techniques, in particular a new generation of apparatus which allow to be performed in the same chamber, studies of capillary properties at high temperatures and chemical analysis of surfaces, a new method to determine wet- ting properties of ceramics by metals based on infiltration studies, and also new methods to measure with improved accuracy the interfacial tension between immiscible liquids, metals, or salts. A number of studies was dedicated to the wettability and joining of special ceramics (e.g, borides, nitrides, and halides), while a renewal of wettability studies in metalmetal systems was observed undoubtedly due to the interest of these systems to the microelectronics industry.

With the attendance of more than 120 scientists from 22 countries, the international flavor of this conference gave a sense of just how geographically broad this highly focused field has become. Moreover, the combination of applied and fundamental research presentations enables participants to understand phenomena, solve problems in processing, and address new questions.

The next HTC conference will be held in Athens, Greece, in the spring of 2009.

ENRIQUE LOUIS, Conference Chair Nicolas Eustathopoulos, Program Chair

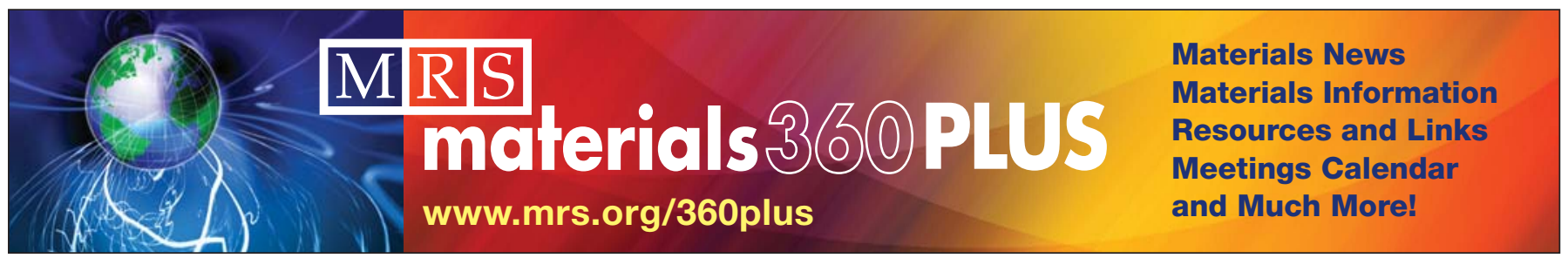

